\title{
Uniqueness for inhomogeneous Dirichlet problem for elliptic-parabolic equations
}

Boris P. Andreianov

Laboratoire de Mathématiques, Université de Franche-Comté, 25030 Besançon, France (boris.andreianov@math.univ-fcomte.fr)

\section{Noureddine Igbida}

LAMFA, CNRS-UMR 6140, Université de Picardie Jules Verne, 33 rue Saint Leu, 80038 Amiens, France

(noureddine.igbida@u-picardie.fr)

(MS received 23 November 2005; accepted 11 October 2006)

We show the $L^{1}$ contraction and comparison principle for weak (and, more generally, renormalized) solutions of the elliptic-parabolic problem $j(v)_{t}-\operatorname{div}(\nabla w+F(w))=$ $f(t, x), w=\varphi(v)$ in $(0, T) \times \Omega \subset \mathbb{R}^{+} \times \mathbb{R}^{N}$ with inhomogeneous Dirichlet boundary datum $g \in L^{2}\left(0, T ; W^{1,2}(\Omega)\right)$ for $w$ (the boundary datum is taken in the sense $w-g \in$

$\left.L^{2}\left(0, T ; H_{0}^{1}(\Omega)\right)\right)$ and initial datum $j_{o} \in L^{1}(\Omega)$ for $j(v)$. Here $\varphi$ and $j$ are non-decreasing, and we assume that $F$ is just continuous.

Our proof consists in doubling of variables in the interior of $\Omega$ as introduced by Carrillo in 1999, and in a careful treatment of the flux term near the boundary of $\Omega$. For the latter argument, the result is restricted to the linear dependence on $\nabla w$ of the diffusion term. The proof allows for a wide class of domains $\Omega$, including, for example, domains of finite perimeter with uniform exterior cone condition or even domains with cracks.

We obtain the corresponding results for the associated stationary problem and discuss on generalization of our technique to the case of nonlinear diffusion operators.

\section{Introduction}

Let $T>0$, and $\Omega \subset \mathbb{R}^{N}$ be a bounded domain. Consider the problem

$$
\begin{aligned}
j(v)_{t}-\operatorname{div} a(w, \nabla w) & =f, & & \\
w & =\varphi(v) & & \text { in } Q=(0, T) \times \Omega, \\
w & =g & & \text { on } \Sigma=(0, T) \times \partial \Omega, \\
\left.j(v)\right|_{t=0} & =j_{o} & & \text { in } \Omega,
\end{aligned}
$$

where $j, \varphi: \mathbb{R} \rightarrow \mathbb{R}$ are continuous non-decreasing functions, and $a: \mathbb{R} \times \mathbb{R}^{N} \rightarrow \mathbb{R}^{N}$ is continuous and monotone in the second variable. Note that the Dirichlet problem for the equation $u_{t}-\operatorname{div} a(w, \nabla w)=f, w \in \beta(u)$, where $\beta$ is an arbitrary maximal monotone graph on $\mathbb{R}$, reduces to $\left(P_{g}\left(f, j_{o}\right)\right.$ ) (see, for example, $[4,9]$ ).

The aim of this paper is to show the uniqueness (of $j(v)$ ) and, more generally, the $L^{1}$ contraction and comparison result for $\left(P_{g}\left(f, j_{o}\right)\right)$ with respect to the data 
$f \in L^{1}(Q)$ and $j_{o} \in L^{1}(\Omega)$. The boundary condition in $\left(P_{g}\left(f, j_{o}\right)\right)$ is understood in the sense $(w-g) \in L^{p}\left(0, T ; W_{0}^{1, p}(\Omega)\right)$; we assume $g \in L^{p}\left(0, T ; W^{1, p}(\Omega)\right)$. For a sufficiently smooth domain $\Omega$, one can consider $g \in L^{p}\left(0, T, W^{1-1 / p}(\partial \Omega)\right)$ and then take an order-preserving extension of $g$ in $L^{p}\left(0, T, W^{1, p}(\Omega)\right)$.

In the main part of the paper, we consider only the quasilinear equation with convection (in this case, $p=2$ ): it is assumed that

$$
a(w, \nabla w)=\nabla w+F(w), \quad \text { where } F: \mathbb{R} \mapsto \mathbb{R}^{N} \text { is continuous. }
$$

Results for more general fluxes are discussed in $\S 7.2$. Since we are not concerned with the problem of existence of weak solutions, we do not require explicitly the usual growth assumptions on the convection $F$.

The uniqueness of weak solutions to $\left(P_{g}\left(f, j_{o}\right)\right)$ is well known in the case in which $a$ is Lipschitz or, more generally, Hölder continuous (of order $\frac{1}{2}$, for fluxes (H0)) with respect to the first argument [1,23]. Note that if $g$ is independent of $t$ and the existence for the stationary problem is known, this kind of result can be obtained somewhat more easily using the tools of the nonlinear semi-group theory, as in [6], where the uniqueness of a mild solution is shown as $g \equiv 0$. Previous results on mild solutions were obtained in $[5,25]$.

A uniqueness result for the homogeneous case $g \equiv 0$ and the flux $a$ of the form (H0) without any assumption on the modulus of continuity of $F$ is given by Kobayasi in [17] (see [4] for a simpler and more general proof). The main tool in this case is Kruzhkov's doubling-of-variables techniques adapted to parabolic problems, as introduced by Carrillo [9]. Carrillo proves the uniqueness of entropy solutions for the general elliptic-parabolic-hyperbolic problem. As shown in $[4,17]$, these solutions coincide with weak solutions for the case in which we are interested. For $g \equiv 0$ and a wide class of fluxes $a(w, \nabla w)$ including simply continuous convections, the uniqueness of renormalized solutions is shown by Carrillo and Wittbold [10] (another approach for renormalized solutions, which works for Lipschitz convections and avoids the doubling of variables in space, is presented in [8]). In $[4,9,10,17]$, the treatment of the homogeneous boundary condition is carried out through Carrillo's elegant choice of test functions. Thus, the technical difficulty of considering boundary traces for which existence is not clear is bypassed (see [24] for the explicit argument in one case where the strong trace is well defined). Unfortunately, it does not seem straightforward to adapt the Carrillo approach to the non-constant Dirichlet boundary condition $g$. Such an adaptation was recently carried out by Ammar et al. [3], for continuous on $\partial \Omega$ boundary data $g$ (more general $g$ are also treated in [3], but in a quite different way).

Uniqueness of entropy solutions for the inhomogeneous Dirichlet problem was first addressed by Mascia et al. [20], for the parabolic-hyperbolic problem. They used the approach of Otto [22] (see also [19]) and Chen and Frid [11,12], an approach which gives sense to the normal trace of the flux on the boundary. The main effort in [20] was made to treat the difficulties due to the possible hyperbolic behaviour of the problem, and the simplifying assumptions that the boundary data are regular and $F$ is Lipschitz continuous have been introduced. Another technique for the same problem, which is particularly useful for analysis of convergence of finite volume schemes, was developed by Michel and Vovelle [21]. Also in [21], regularity 
assumptions on boundary data and on $F$ were required. Note that, for the purely hyperbolic problem, the general uniqueness result was achieved by Ammar et al. [3].

In the present paper, we give a proof of uniqueness of weak solutions for the inhomogeneous Dirichlet problem for the elliptic-parabolic equation $\left(P_{g}\left(f, j_{o}\right)\right)$ with the flux (H0), without the Lipschitz or Hölder assumptions on the convection term, and for a wide class of domains. We first perform the standard doubling of variables in the interior of $\Omega$ (see $[4,9,15,17]$ ). In order to generate the boundary term, we consider test functions that truncate in a neighbourhood of $\partial \Omega$, in the spirit of [24], [20, see in particular remark 1.5], [17] and [4]. Under some mild assumptions on $\partial \Omega$ ( see (H1) and (H2) in $\S 2$ ), we construct the test functions such that the 'boundary' term coming from the comparison of two solutions has a sign, the fact which was implicit in the Carrillo argument. Note that our argument requires the flux $a(w, \nabla w)$ to be linear in $\nabla w$; its generalization to, for example, diffusions of the $p$-Laplacian type $|\nabla w|^{p-2} \nabla w$ is an open question (see $\S 7.2$ ).

The paper is organized as follows. In $\S 2$, we give definitions and state the results for the evolution problem. In $\S 3$, we deduce the corresponding results for the associated stationary problem. In $\S 4$ we sketch the doubling-of-variables argument in the interior of $\Omega$ (see [4] and the references therein for a more detailed exposition). The explicit treatment of the boundary terms is carried out in $\S 5$. The proof of the main result is contained in $\S 6$. In $\S 7$, we prove the $L^{1}$ contraction and comparison principle for renormalized solutions of $\left(P_{g}\left(f, j_{o}\right)\right)$ (for related works, see $[2,7,8,10,16]$ and references therein). Then we discuss extensions of theorems 2.3 and 3.2 to more general elliptic-parabolic problems.

\section{Definitions and results}

Definition 2.1. Let $p \in(1,+\infty), p^{\prime}=p /(p-1)$ and $g \in L^{p}\left(0, T ; W^{1, p}(\Omega)\right)$. An almost everywhere (a.e.) defined measurable function $v: Q \mapsto \overline{\mathbb{R}}$ is called a weak solution of $\left(P_{g}\left(f, j_{o}\right)\right)$ if $j(v) \in L^{1}(\Omega)$, the function $w=\varphi(v)$ satisfies $w \in$ $g+L^{p}\left(0, T ; W_{0}^{1, p}(\Omega)\right)$ and $a(w, \nabla w) \in L^{p^{\prime}}(Q)^{N}$, and the distributional derivative $j(v)_{t}$ can be identified with $\chi \in L^{p^{\prime}}\left(0, T ; W^{-1, p}(\Omega)\right)+L^{1}(Q)$ such that

$$
\int_{0}^{T}\langle\chi, \xi\rangle+\iint_{Q} a(w, \nabla w) \cdot \nabla \xi=\iint_{Q} f \xi
$$

for all test functions $\xi \in L^{p}\left(0, T ; W_{0}^{1, p}(\Omega)\right) \cap L^{\infty}(Q)$, and

$$
\int_{0}^{T}\langle\chi, \xi\rangle=-\iint_{Q} j(v) \xi_{t}-\int_{\Omega} j_{o}(x) \xi(0, x)
$$

for all test functions $\xi \in L^{p}\left(0, T ; W_{0}^{1, p}(\Omega)\right) \cap L^{\infty}(Q)$ such that $\xi_{t} \in L^{\infty}(Q)$ and $\xi(T, \cdot)=0$.

Here and throughout the paper, we denote by $\langle\cdot, \cdot\rangle$ the duality pairing between $W_{0}^{1, p}(\Omega) \cap L^{\infty}(\Omega)$ and its dual. Except for in $\S 7.2$, we will always assume $p=2$ and write $H_{0}^{1}$ for $W_{0}^{1,2}$.

In order to formulate the assumptions on $\Omega$, we require that $\Omega \supset \tilde{\Omega}$ for all open $\tilde{\Omega} \subset \bar{\Omega}$ such that $H_{0}^{1}(\tilde{\Omega})=H_{0}^{1}(\Omega)$. Assume that

$$
\text { the }(N-1) \text {-dimensional Hausdorff measure of } \partial \Omega \text { is finite. }
$$


Assume the following Poincaré-Friedrichs property.

(H2) There exists a constant $\mathcal{M}$, independent of $h$, such that, for all $x_{o} \in \partial \Omega$ and all $W \in H_{0}^{1}(\Omega)$, we have

$$
\int_{B_{h}\left(x_{o}\right) \cap \Omega}|W|^{2} \leqslant \mathcal{M} h \int_{B_{h}\left(x_{o}\right) \cap \Omega}|\nabla W|^{2} .
$$

Here $B_{h}\left(x_{o}\right)$ denotes the $N$-dimensional ball of radius $h$ centred at $x_{o}$.

REMARK 2.2. It is easy to see that $(\mathrm{H} 2)$ is verified in the case when $\Omega$ is weakly Lipschitz (that is, each point $x \in \partial \Omega$ possesses a neighbourhood $U_{x}$ such that $\Omega \cap U_{x}$ can be mapped on a half-ball of $\mathbb{R}^{n}$ by a bi-Lipschitz homeomorphism).

More generally, assume that, for $d=N$, we have

$$
\inf _{h>0, x_{o} \in \partial \Omega} \frac{1}{h^{d}}\left|B_{h}\left(x_{o}\right) \backslash \Omega\right|>0,
$$

where $|\cdot|$ denotes the $N$-dimensional Lebesgue measure. Then (H2) holds (see, for example, [26, theorem 3.11.1]). Note that, for instance, the uniform exterior cone condition implies the condition $\left(\mathrm{H} 2^{\prime}(N)\right)$, and thus $(\mathrm{H} 2)$.

In both the aforementioned cases, the inequality in (H2) actually holds with $h$ replaced by $h^{2}$. A sharper condition sufficient for (H2) to hold can be formulated in terms of the Bessel capacity $B_{1,2}$ (see, for example, [26]):

$$
\inf _{h>0, x_{o} \in \partial \Omega} \frac{1}{h} B_{1,2}\left(\mathcal{N}_{h}\left(x_{o}\right)\right)>0, \quad \text { where } \mathcal{N}_{h}\left(x_{o}\right)=\left\{\frac{1}{h}\left(x-x_{o}\right) \mid x \in B_{h}\left(x_{o}\right) \backslash \Omega\right\} .
$$

This condition permits us, in particular, to include domains with cracks. For the proof, it is sufficient to map $B_{h}\left(x_{o}\right)$ on the unit ball of $\mathbb{R}^{N}$ and apply [26, corollary 4.5.3].

For $N \geqslant 3, B_{1,2}\left(\mathcal{N}_{h}\left(x_{o}\right)\right)$ in $(2.4)$ can be replaced by the Newtonian capacity of $\mathcal{N}_{h}\left(x_{o}\right)$ in $\mathbb{R}^{N}$ (cf. [26, exercise 2.8]) and then by

$$
\left|\mathcal{N}_{h}\left(x_{o}\right)\right|^{(N-2) / N}=\frac{1}{h^{N-2}}\left|B_{h}\left(x_{o}\right) \backslash \Omega\right|^{(N-2) / N}
$$

(see, for example, [13, theorem 4.7.2]). Thus, (H2) still holds, if $N \geqslant 3$ and the aforementioned condition $\left(\mathrm{H}_{2}^{\prime}(d)\right)$ is fulfilled with $d=N(N-1) /(N-2)$.

Let us state the main result of this paper. Denote by $\operatorname{sgn}^{+}(\cdot)$ the maximal monotone extension of the function

$$
\operatorname{sgn}_{0}^{+}: r \in \mathbb{R} \mapsto \begin{cases}0, & r \leqslant 0 \\ 1, & r>0\end{cases}
$$

THEOREM 2.3. Assume that (H1) and (H2) hold. Let $v$ and $\hat{v}$ be weak solutions of $P_{g}\left(f, j_{o}\right)$ and $\left(P_{\hat{g}}\left(\hat{f}, \hat{\jmath}_{o}\right)\right)$, respectively, with the flux given by (HO) and $p=2$. Assume $g \leqslant \hat{g}$. Then there exists $\eta: Q \rightarrow \mathbb{R}, \eta \in \operatorname{sgn}^{+}(j(v)-j(\hat{v}))$ a.e. on $Q$, such that, for a.e. $t \in(0, T)$, we have

$$
\int_{\Omega}(j(v)-j(\hat{v}))^{+}(t) \leqslant \int_{\Omega}\left(j_{o}-\hat{\jmath}_{o}\right)^{+}+\int_{0}^{t} \int_{\Omega} \eta(f-\hat{f}) .
$$


In particular, if $\hat{g} \geqslant g, \hat{f} \geqslant f$ a.e. on $Q$ and $\hat{\jmath}_{o} \geqslant j_{o}$ a.e. on $\Omega$, then $j(\hat{v}) \geqslant j(v)$ a.e. on $Q$.

The uniqueness result for $j(v)$ follows readily.

Corollary 2.4. Assume (HO), (H1) and (H2) hold. For all $g \in L^{2}\left(0, T, W^{1,2}(\Omega)\right)$, $f \in L^{1}(Q)$ and $j_{o} \in L^{1}(\Omega)$, there exists at most one function $j(v) \in L^{1}(Q)$ such that $v$ is a weak solution to $\left(P_{g}\left(f, j_{o}\right)\right)$. In particular, if $j$ is injective, there exists at most one weak solution $v$ to $\left(P_{g}\left(f, j_{o}\right)\right)$.

\section{The stationary problem}

We also consider weak solutions to the associated 'stationary' elliptic problem with $f \in L^{1}(\Omega)$ :

$$
\left.\begin{array}{rl}
j(v)-\operatorname{div} a(w, \nabla w) & =f, \quad w=\varphi(v) \quad \text { in } \Omega, \\
w & =g \quad \text { on } \partial \Omega
\end{array}\right\}
$$

in the sense of the following definition.

Definition 3.1. Let $p \in(1,+\infty), p^{\prime}=p /(p-1)$, and $g \in W^{1, p}(\Omega)$. An a.e. defined measurable function $v: \Omega \mapsto \overline{\mathbb{R}}$ is called a weak solution of $\left(S_{g}(f)\right)$ if $j(v) \in L^{1}(\Omega)$, the function $w=\varphi(v)$ is such that $w \in g+W_{0}^{1, p}(\Omega)$ and $a(w, \nabla w) \in L^{p^{\prime}}(\Omega)^{N}$, and

$$
\int_{\Omega} j(v) \xi+\iint_{Q} a(w, \nabla w) \cdot \nabla \xi=\iint_{Q} f \xi
$$

for all test functions $\xi \in W_{0}^{1, p}(\Omega) \cap L^{\infty}(\Omega)$.

TheOREM 3.2. Assume (H1) and (H2) hold. Let $v$ and $\hat{v}$ be weak solutions of $\left(S_{g}(f)\right)$ and $\left(S_{\hat{g}}(\hat{f})\right)$, respectively, with the flux given by $(H O)$ and $p=2$. Assume $g \leqslant \hat{g}$. Then there exists $\eta: \Omega \rightarrow \mathbb{R}, \eta \in \operatorname{sgn}^{+}(j(v)-j(\hat{v}))$ a.e. on $\Omega$, such that

$$
\int_{\Omega}(j(v)-j(\hat{v}))^{+} \leqslant \int_{\Omega} \eta(f-\hat{f}) .
$$

In particular, if $\hat{g} \geqslant g$ and $\hat{f} \geqslant f$ a.e. on $\Omega$, then $j(\hat{v}) \geqslant j(v)$ a.e. on $\Omega$.

Proof. Let $v$ be a weak solution of $\left(S_{g}(f)\right)$. Set $\tilde{v}(t) \equiv v$; then $\tilde{v}$ is a weak solution of $\left(P_{g}\left(\tilde{f}, \tilde{\jmath}_{o}\right)\right)$ corresponding to the data $\tilde{\jmath}_{o}=j(v)$ and $\tilde{f}=f-v$. Hence, the result follows readily by theorem 2.3 .

The corresponding uniqueness result for $j(v)$ follows.

Corollary 3.3. Assume (HO), (H1) and (H2) hold. For all $g \in W^{1,2}(\Omega)$ and $f \in L^{1}(\Omega)$, there exists at most one function $j(v) \in L^{1}(\Omega)$ such that $v$ is a weak solution to $\left(S_{g}(f)\right)$. In particular, if $j$ is injective, there exists at most one weak solution $v$ to $\left(S_{g}(f)\right)$.

Note that, for the stationary problem, we are able to extend theorem 3.2 to nonlinear fluxes of the form $a(w, \nabla w)=b(\nabla w)+F(w)$, in case $p=2$ and under an additional structure assumption on $b: \mathbb{R}^{N} \rightarrow \mathbb{R}^{N}$ (see $\S 7.2$ ). 


\section{The doubling of variables}

Let $v, \hat{v}$ be two weak solutions of $\left(P_{g}\left(f, j_{o}\right)\right)$ and $\left(P_{\hat{g}}\left(\hat{f}, \hat{\jmath}_{o}\right)\right)$, respectively. We have the following comparison principle in the interior of $\Omega$.

Lemma 4.1. Let $v$ and $\hat{v}$ be the two weak solutions in theorem 2.3; let $w=\varphi(v)$, $\hat{w}=\varphi(\hat{v})$. There exists $\eta: Q \rightarrow \mathbb{R}, \eta \in \operatorname{sgn}^{+}(j(v)-j(\hat{v}))$ a.e. on $Q$, such that, for all $\xi \in H_{0}^{1}(\Omega)$, for a.e. $t \in(0, T)$, we have

$$
\begin{aligned}
& \int_{\Omega}(j(v)(t)-j(\hat{v})(t))^{+} \xi-\int_{\Omega}\left(j_{o}-\hat{\jmath}_{o}\right)^{+} \xi-\int_{0}^{t} \int_{\Omega} \eta(f-\hat{f}) \xi \\
& \quad \leqslant-\int_{0}^{t} \int_{\Omega} \nabla(w-\hat{w})^{+} \cdot \nabla \xi-\int_{0}^{t} \int_{\Omega} \operatorname{sgn}_{0}^{+}(w-\hat{w})(F(w)-F(\hat{w})) \cdot \nabla \xi .
\end{aligned}
$$

In order to prove lemma 4.1, one needs the 'entropy inequalities' (4.2) below.

Lemma 4.2. Let $\xi \in H_{0}^{1}(\Omega)$ and let $v$ be a weak solution of $P_{g}\left(f, j_{o}\right)$; then

$$
\begin{array}{r}
\iint_{Q}-(j(v)-j(k))^{+} \xi \psi_{t}-\int_{\Omega} \xi \psi(0)\left(j_{o}-j(k)\right)^{+}-\iint_{Q} f \xi \psi \operatorname{sgn}_{0}^{+}(v-k) \\
\leqslant-\iint_{Q}(\nabla \varphi(v)+F(\varphi(v))-F(\varphi(k))) \cdot \nabla \xi \psi \operatorname{sgn}_{0}^{+}(v-k)
\end{array}
$$

for all $k \in \mathbb{R}$ and all $\psi \in \mathcal{D}(-\infty, T)$.

The outline of the proof of lemma 4.2 is given in [4, lemmas 1 and 2]. The original proofs can be found in $[9,15,17]$. We omit the details in order to avoid the unnecessary duplication of arguments.

Proof of lemma 4.1. First fix $\xi \in \mathcal{D}(\Omega)$. Take any Lipschitz domain $B$ of $\mathbb{R}^{N}$ such that $\operatorname{supp} \xi \subset B \subset \Omega$ (we can always choose $B$ polygonal). Using the entropy inequalities (4.2) applied on $B$ and Carrillo's adaptation of the Kruzhkov doublingof-variables method (cf. [9, theorem 9] and [18], respectively), we deduce (4.1). The result for general $\xi \in H_{0}^{1}(\Omega)$ follows by the density argument.

\section{An approach for the boundary flux}

Note that, heuristically, the limit, as $\xi$ converges to 1 on $\Omega$, of the right-hand side of (4.1) should be the boundary term

$$
\int_{0}^{t} \int_{\partial \Omega} \frac{\partial}{\partial n}(w-\hat{w})^{+}+\int_{0}^{t} \int_{\partial \Omega} \operatorname{sgn}_{0}^{+}(w-\hat{w})(F(w)-F(\hat{w})) \cdot n,
$$

where $n$ denotes the exterior unit normal to $\partial \Omega$. Moreover, if (5.1) can be understood in the pointwise sense (e.g. for $\Omega$ and $w, \hat{w}$ regular enough), then it is nonpositive since $(w-\hat{w})^{+} \geqslant 0$ in $\Omega$ and $(w-\hat{w})^{+}=0$ on $\partial \Omega$, within the assumptions of theorem 2.3 .

In this section, we search for test functions $\xi_{h}, h>0$, such that $\xi_{h} \rightarrow 1$ as $h \rightarrow 0$ a.e. on $\Omega$, and that would allow us to pass to the limit on the right-hand side of (4.1), generating non-positive 'boundary' terms. In the rest of the paper we 
denote by $\mathcal{M}$ a generic constant that may depend on $w, \hat{w}, \Omega, T$, on coefficients and the data of the problem, but that is independent of $h$.

\subsection{Assumptions on $\Omega$}

Denote by $\Omega_{h}$ the $h$-neighbourhood of $\partial \Omega$ in $\Omega: \Omega_{h}=\{x \in \Omega \mid \operatorname{dist}(x, \partial \Omega)<$ $h\}$. Denote by $\left|\Omega_{h}\right|$ its $N$-dimensional Lebesgue measure. We need the following assumptions on $\Omega$.

$\left(\mathrm{H}^{\Omega}\right)$ There exists a constant $\mathcal{M}$, independent of $h$, such that

(i) $\left|\Omega_{h}\right| \leqslant \mathcal{M} h$ for all $h$ sufficiently small,

(ii) the following Poincaré-Friedrichs inequality in $\Omega_{h}$ holds:

$$
\int_{\Omega_{h}}|W|^{2} \leqslant \mathcal{M} h \int_{\Omega_{4 h}}|\nabla W|^{2}, \quad \text { for all } W \in H_{0}^{1}(\Omega) .
$$

Note that introducing $\Omega_{4 h}$ in $\left(\mathrm{H}^{\Omega}\right)$ (ii) is due only to the convenience of stating (H2) for balls $B_{h}\left(x_{o}\right)$, rather than for general convex neighbourhoods of $x_{o}$.

The assumptions (H1) and (H2) given in $\S 2$ are sufficient for (i) and (ii) of $\left(\mathrm{H}^{\Omega}\right)$ to hold. More exactly, we have the following lemma.

LEMma 5.1.

(i) Assume (H1) holds. Then (i) of $\left(H^{\Omega}\right)$ is true.

(ii) Assume (H2) holds. Then (ii) of $\left(H^{\Omega}\right)$ is true.

Proof. (i) Take a countable covering $\mathcal{C}$ of $\partial \Omega$ by balls $B_{i}$ of radii $r_{i}, r_{i}<h$; the balls with the same centres and of radii $2 r_{i}$ cover $\Omega_{h}$. Hence, $\left|\Omega_{h}\right| \leqslant \sum_{B_{i} \in \mathcal{C}} c_{N}\left(2 r_{i}\right)^{N}$, where $c_{N}$ is the measure of the unit ball of $\mathbb{R}^{N}$. By definition,

$$
\lim _{h \rightarrow 0} \inf _{\mathcal{C}} c_{N} \sum_{B_{i} \in \mathcal{C}} r_{i}^{N-1}
$$

is equal to $\mathcal{H}^{N-1}(\partial \Omega)$, up to a normalizing factor. We deduce that $\left|\Omega_{h}\right| \leqslant \mathcal{M} h$.

(ii) Take a finite covering $\left\{B_{2 h}\left(x_{i}\right)\right\}$ of $\partial \Omega$ by balls of radius $2 h$ centred at points $x_{i} \in \partial \Omega$. The balls with the same centres and of radius $4 h$ cover $\Omega_{h}$; moreover, if $\operatorname{dist}\left(x_{i}, x_{j}\right)<h$, we can omit one of the balls $B_{4 h}\left(x_{i}\right), B_{4 h}\left(x_{j}\right)$ in this covering. This implies that each point of $\Omega_{h}$ belongs to at most $\mathcal{L}$ different balls $B_{4 h}\left(x_{i}\right)$, with an $\mathcal{L}$ that depends only on the dimension $N$. Applying $(\mathrm{H} 2)$ to each of $B_{4 h}\left(x_{i}\right)$, we get (ii) of $\left(\mathrm{H}^{\Omega}\right)$ with $\mathcal{M}$ replaced by $\mathcal{L} \mathcal{M}$.

\subsection{Construction of $\xi_{h}$}

Lemma 5.2. Assume $\left(H^{\Omega}\right)$ holds. Let $w, \hat{w} \in L^{2}\left(0, T ; W^{1,2}(\Omega)\right)$ such that $(w-$ $\hat{w})^{+} \in L^{2}\left(0, T, H_{0}^{1}(\Omega)\right)$. Assume in addition that $w, \hat{w} \in L^{\infty}(Q)$. Then there exists a sequence $\left(\xi_{h_{m}}\right)_{m \in \mathbb{N}} \subset H_{0}^{1}(\Omega)$ such that $0 \leqslant \xi_{h_{m}} \leqslant 1$ and $\xi_{h_{m}} \rightarrow 1$ as $m \rightarrow \infty$ a.e. 
on $\Omega$, and

$$
\begin{gathered}
\lim _{m \rightarrow \infty} \int_{0}^{t} \int_{\Omega} \nabla(w-\hat{w})^{+} \cdot \nabla \xi_{h_{m}} \geqslant 0 \quad \text { for all } t \in(0, T), \\
\lim _{m \rightarrow \infty} \iint_{Q} \operatorname{sgn}_{0}^{+}(w-\hat{w})|F(w)-F(\hat{w})|\left|\nabla \xi_{h_{m}}\right|=0 .
\end{gathered}
$$

Lemma 5.2 is a direct consequence of lemmas 5.4 and 5.5 below. In lemma 5.4 , we give a construction ensuring (5.3) together with the additional properties (5.5), which are needed for the proof of (5.4) given in lemma 5.5.

REMARK 5.3. Note that, as a straightforward choice, one could take for $\xi_{h}$ the distance-to-the-boundary functions $\xi_{h}^{o}=h^{-1} \min \{h, \operatorname{dist}(x, \partial \Omega)\}$. Proving lemma 5.2 with $\xi_{h}^{o}$ seems to require more smoothness on $\partial \Omega$. See remark 7.5 for a further discussion of this issue.

LEMMA 5.4. There exists a family $\left(\xi_{h}\right)_{h>0} \in H_{0}^{1}(\Omega)$ and a constant $\mathcal{M}>0$ such that

(i) $0 \leqslant \xi_{h} \leqslant 1$, and $\xi_{h} \rightarrow 1$ a.e. in $\Omega$ as $h \rightarrow 0$,

(ii) for all positive $W \in H_{0}^{1}(\Omega), \int_{\Omega} \nabla W \cdot \nabla \xi_{h} \geqslant 0$,

(iii) under assumption (i) of $\left(H^{\Omega}\right)$, we have

$$
\frac{1}{\mathcal{M}} \leqslant \int_{\Omega}\left|\nabla \xi_{h}\right| \leqslant \mathcal{M}, \quad \int_{\Omega}\left|\nabla \xi_{h}\right|^{2} \leqslant \frac{\mathcal{M}}{h}, \quad \operatorname{supp} \nabla \xi_{h} \subset \Omega_{h} .
$$

Proof. For sufficiently small $h$ and $x \in \Omega_{h}$, set $u_{h}^{o}(x)=\operatorname{dist}(x, \partial \Omega)$. Denote by $u_{h}^{*}$ the variational solution $u \in u_{h}^{o}+H_{0}^{1}(\Omega)$ of the problem

$$
\left.\begin{array}{rl}
\Delta u=0 & \text { in } \Omega_{h}, \\
u=u_{h}^{o} & \text { on } \partial \Omega_{h} .
\end{array}\right\}
$$

Set

$$
\xi_{h}=\frac{2}{h} \min \left\{u_{h}^{*}, \frac{h}{2}\right\} .
$$

Extending $\xi_{h}$ by the value 1 on $\Omega \backslash \Omega_{h}$, we see that (i) holds.

Let us show (iii). By construction, supp $\nabla \xi_{h} \subset \Omega_{h}$. For $h$ sufficiently small, the Friedrichs inequality yields the lower bound on $\int_{\Omega}\left|\nabla \xi_{h}\right|$ :

$$
\int_{\Omega}\left|\nabla \xi_{h}\right| \geqslant \frac{1}{\mathcal{M}} \int_{\Omega}\left|\xi_{h}\right| \geqslant \frac{|\Omega|}{2 \mathcal{M}}
$$

Finally, we have $\left|\nabla u_{h}^{o}\right| \leqslant 1$ in $\Omega_{h}$, because $u_{h}^{o}$ is Lipschitz continuous with the Lipschitz constant equal to 1 . Using the variational interpretation of (5.6), the 
Hölder inequality and (i) of $\left(\mathrm{H}^{\Omega}\right)$, we deduce that

$$
\begin{gathered}
\int_{\Omega}\left|\nabla \xi_{h}\right|^{2} \leqslant \frac{4}{h^{2}} \int_{\Omega_{h}}\left|\nabla u_{h}^{*}\right|^{2} \leqslant \frac{4}{h^{2}} \int_{\Omega_{h}}\left|\nabla u_{h}^{o}\right|^{2} \leqslant \frac{4\left|\Omega_{h}\right|}{h^{2}} \leqslant \frac{\mathcal{M}}{h}, \\
\int_{\Omega}\left|\nabla \xi_{h}\right| \leqslant \int_{\Omega_{h}}\left|\nabla \xi_{h}\right| \leqslant\left(\left|\Omega_{h}\right| \int_{\Omega_{h}}\left|\nabla \xi_{h}\right|^{2}\right)^{1 / 2} \leqslant \mathcal{M} .
\end{gathered}
$$

Let us show that (ii) holds. Denote $\Omega_{h}^{*}=\left\{x \in \Omega_{h} \mid u_{h}^{*}<\frac{1}{2}\right\}$, and $\partial^{\text {int }} \Omega_{h}^{*}=$ $\partial \Omega_{h}^{*} \backslash \partial \Omega ; \partial^{\text {int }} \Omega_{h}^{*}$ is the $\frac{1}{2}$-level set of $u_{h}^{*}$. Let $n$ denote the exterior unit normal vector to $\partial^{\text {int }} \Omega_{h}^{*}$. By the interior regularity result for the problem (5.6), it follows that $\left.\nabla u_{h}^{*} \cdot n\right|_{\partial^{\text {int }} \Omega_{h}^{*}}$ exists in the pointwise sense; moreover, it is non-negative, by definition of $\Omega_{h}^{*}$. Thus, $\xi_{h}$ solves the Laplace equation in $\Omega_{h}^{*}$ with the zero Dirichlet condition on $\partial \Omega$ and a non-negative Neumann condition on $\partial^{\text {int }} \Omega_{h}^{*}$. Taking $W$ as a test function, we get

$$
0 \leqslant \int_{\partial^{\text {int }} \Omega_{h}^{*}} W \nabla \xi_{h} \cdot n=\int_{\Omega_{h}^{*}} \nabla W \cdot \nabla \xi_{h}=\int_{\Omega} \nabla W \cdot \nabla \xi_{h} .
$$

Lemma 5.5. Assume (ii) of $\left(H^{\Omega}\right)$ holds. Let $\left(\xi_{h}\right)_{h>0} \subset H_{0}^{1}(\Omega)$ be a family of functions satisfying (5.5), and $h_{m}=1 / m$. Then (5.4) holds.

Proof. Set $M=\max \left\{\|w\|_{\infty},\|\hat{w}\|_{\infty}\right\}$. There exists a continuous concave function $\Psi:[0,2 M] \rightarrow \mathbb{R}^{+}, \Psi(0)=0$, such that

$$
|F(z)-F(\hat{z})| \leqslant \Psi(r) \quad \text { for all } z, \hat{z} \in \mathbb{R} \text { with }|z-\hat{z}| \leqslant r \text { and }|z|,|\hat{z}| \leqslant M
$$

Using (5.7) and applying the Jensen inequality for $\Psi$ with respect to the measure on $Q$ given by $\left|\nabla \xi_{h}\right| \mathrm{d} x \mathrm{~d} t$, we get

$$
\begin{aligned}
\iint_{Q} \operatorname{sgn}_{0}^{+}(w-\hat{w})|F(w)-F(\hat{w})|\left|\nabla \xi_{h_{m}}\right| \\
\leqslant \iint_{Q} \Psi\left((w-\hat{w})^{+}\right)\left|\nabla \xi_{h_{m}}\right| \\
\leqslant\left(\iint_{Q}\left|\nabla \xi_{h}\right|\right) \Psi\left(\frac{1}{\iint_{Q}\left|\nabla \xi_{h}\right|} \iint_{Q}(w-\hat{w})^{+}\left|\nabla \xi_{h}\right|\right) .
\end{aligned}
$$

Denote the left-hand side of (5.8) by $I_{h}$. Using the Cauchy-Schwarz inequality and the properties (5.5), we deduce

$$
I_{h} \leqslant \mathcal{M} \Psi\left(\mathcal{M}\left(\frac{1}{h} \iint_{(0, T) \times \Omega_{h}}\left|(w-\hat{w})^{+}\right|^{2}\right)^{1 / 2}\right) .
$$

Finally, note that since $g \leqslant \hat{g}$ and $w-g, \hat{w}-\hat{g} \in L^{2}\left(0, T, H_{0}^{1}(\Omega)\right)$, we have $(w-$ $\hat{w})^{+}(t) \in H_{0}^{1}(\Omega)$ for a.e. $t \in(0, T)$. Hence, the Poincaré-Friedrichs inequality (ii) of $\left(\mathrm{H}^{\Omega}\right)$ yields

$$
I_{h} \leqslant \mathcal{M} \Psi\left(\mathcal{M}^{3 / 2}\left(\iint_{(0, T) \times \Omega_{4 h}}|\nabla(w-\hat{w})|^{2}\right)^{1 / 2}\right) .
$$

Thus, $I_{h}$ converges to zero as $h \rightarrow 0$, which ends the proof. 


\section{Proof of theorem 2.3}

\subsection{The $L^{\infty}$ case}

Assume that $w$ and $\hat{w}$ are bounded on $Q$. Then the claim of the theorem follows readily by lemmas $4.1,5.1,5.2$ and the dominated convergence theorem.

\subsection{The general case}

Let us reduce the general case to that in $\S 6.1$. We proceed as in [16], taking advantage of the homogeneity of the (linear) diffusion term $\operatorname{div} \nabla w$. Let $\left(S_{M}\right)_{M \in \mathbb{N}}$ be a sequence of $\mathcal{C}^{1}(\mathbb{R}, \mathbb{R})$ functions such that $S_{M}(z)=1$ for $|z| \leqslant M-1, S_{M}(z)=0$ for $|z| \geqslant M$, and $\max _{\mathbb{R}}\left|S_{M}^{\prime}\right| \leqslant 2$. Let $v$ be a weak solution to $\left(P_{g}\left(f, j_{o}\right)\right), w=\varphi(v)$. Let $v_{o}: \Omega \rightarrow \mathbb{R}$ be a measurable function such that $j\left(v_{o}\right)=j_{o}$. Let us define the functions

$$
\begin{gathered}
j_{M}(r)=\int_{0}^{r} S_{M}(\varphi(z)) \mathrm{d} j(z), \quad j_{M, o}=j_{M}\left(v_{o}\right), \quad \varphi_{M}(r)=\int_{0}^{\varphi(r)} S_{M}(z) \mathrm{d} z, \\
g_{M}=\int_{0}^{g} S_{M}(z) \mathrm{d} z, \quad f_{M}=f S_{M}(w)-((\nabla w+F(w)) \cdot \nabla w) S_{M}^{\prime}(w), \\
F_{M}=\left(F S_{M}\right) \circ H, \quad \text { where } H(r)= \begin{cases}\min \left\{s \mid \int_{0}^{s} S_{M}(z) \mathrm{d} z=r\right\}, & r \geqslant 0, \\
\max \left\{s \mid \int_{0}^{s} S_{M}(z) \mathrm{d} z=r\right\}, & r \leqslant 0 .\end{cases}
\end{gathered}
$$

Note that we actually have $F_{M}\left(w_{M}\right)=F(w) S_{M}(w)$, and $F_{M}$ is a correctly defined continuous function. Furthermore, $g_{M} \in L^{2}\left(0, T ; W^{1,2}(\Omega)\right)$ and

$$
\left|w_{M}-g_{M}\right|=\left|\int_{g}^{w} S_{M}(z) \mathrm{d} z\right| \leqslant|w-g|,
$$

so that $w_{M}-g_{M} \in L^{2}\left(0, T ; H_{0}^{1}(\Omega)\right)$. Note that $\hat{g} \geqslant g$ implies $\hat{g}_{M} \geqslant g_{M}$. Finally, $f_{M} \rightarrow f$ in $L^{1}(Q)$ and $j_{M, o} \rightarrow j_{o}$ in $L^{1}(\Omega)$ as $M \rightarrow \infty$, by the dominated convergence theorem.

Take an admissible test function $\xi$ in definition 2.1. Then

$$
\xi \frac{1}{h} \int_{t}^{t+h} S_{M}(w)
$$

is still an admissible test function. Passing to the limit as $h \rightarrow 0$, by the chain rule lemma (see [1] for the version we use; see also [10,23]), we find that $v$ is also a weak solution to the auxiliary problem

$$
\begin{aligned}
j_{M}(v)_{t}-\operatorname{div}\left(\nabla w_{M}+F_{M}\left(w_{M}\right)\right) & =f_{M}(t, x), & & \\
w_{M} & =\varphi_{M}(v) & & \text { in } Q=(0, T) \times \Omega, \\
w_{M} & =g_{M} & & \text { on } \Sigma=(0, T) \times \partial \Omega, \\
\left.j_{M}(v)\right|_{t=0} & =j_{M, o} & & \text { in } \Omega .
\end{aligned} \quad\left(P_{g_{M}}^{M}\left(f_{M}, j_{M, o}\right)\right)
$$


(Formally, this point of view corresponds to multiplying the equation in $\left(P_{g}\left(f, j_{o}\right)\right)$ by $S_{M}(w)$.)

Now we have $\left|w_{M}\right| \leqslant M$. Applying the same construction to the solution $\hat{v}$, we find ourselves in the $L^{\infty}$ case for the problems $\left(P_{g_{M}}^{M}\left(f_{M}, j_{M, o}\right)\right)$ and $\left(P_{\hat{g}_{M}}^{M}\left(\hat{f}_{M}, \hat{\jmath}_{M, o}\right)\right)$. Thus, (2.5) holds with $f, \hat{f}$ and $j, j_{o}, \hat{\jmath}_{o}$ replaced by $f_{M}, \hat{f}_{M}$ and $j_{M}, j_{M, o}, \hat{\jmath}_{M, o}$, respectively. As $M \rightarrow \infty$, by the dominated convergence theorem we deduce the claim of the theorem.

\section{Generalizations}

Let us give different extensions of our results and discuss the limitations of our techniques.

\subsection{Extension to renormalized solutions}

The reduction to the case $w \in L^{\infty}(Q)$ used in the proof of theorem 2.3 is inspired by the technique of Igbida and Wittbold [16] developed for renormalized solutions. In this section, we further use it in order to extend the result of theorem 2.3 to renormalized solutions of $\left(P_{g}\left(f, j_{o}\right)\right)$. Note that the result of theorem 3.2 adapts to the case of renormalized solutions of the stationary problem $\left(S_{g}(f)\right)$ in the same way.

Let us first recall the notion of renormalized solutions of elliptic-parabolic problems (see e.g. $[7,8,10]$ for the motivation). For $k>0$ we denote by $T_{k}$ the truncation function defined by $T_{k}: r \in \mathbb{R} \mapsto \operatorname{sgn} r \min \{k,|r|\}$.

Definition 7.1. Let $p \in(1,+\infty), p^{\prime}=p /(p-1)$. Assume $\left(f, g, j_{o}\right) \in L^{1}(Q) \times$ $L^{1}(Q) \times L^{1}(\Omega)$, and $T_{k}(g) \in L^{2}\left(0, T ; W^{1, p}(\Omega)\right)$ for all $k>0$. An a.e. defined measurable function $v: Q \mapsto \overline{\mathbb{R}}$ is called a renormalized solution of $\left(P_{g}\left(f, j_{o}\right)\right)$ if $j(v) \in L^{1}(\Omega)$, the function $w=\varphi(v)$ is such that $T_{k}(w) \in L^{p}\left(0, T ; W^{1, p}(\Omega)\right)$ with $T_{k}(w-g) \in L^{p}\left(0, T ; W_{0}^{1, p}(\Omega)\right), a\left(w, \nabla T_{k}(w)\right) \in L^{p^{\prime}}(Q)^{N}$ and

(i) for any compactly supported $S \in \mathcal{C}^{1}(\mathbb{R} ; \mathbb{R})$ the distributional derivative

$$
\left(\int_{0}^{j(v)} S(z) \mathrm{d} z\right)_{t}
$$

can be identified with $\chi_{S} \in L^{p^{\prime}}\left(0, T ; W^{-1, p^{\prime}}(\Omega)\right)+L^{1}(Q)$ such that

$$
\int_{0}^{T}\left\langle\chi_{S}, \xi\right\rangle+\iint_{Q} a(w, \nabla w) \cdot \nabla(S(w) \xi)=\iint_{Q} f S(w) \xi
$$

for all test functions $\xi \in L^{p}\left(0, T ; W_{0}^{1, p}(\Omega)\right) \cap L^{\infty}(Q)$, and

$$
\int_{0}^{T}\left\langle\chi_{S}, \xi\right\rangle=-\iint_{Q}\left(\int_{0}^{j(v)} S(z) \mathrm{d} z\right) \xi_{t}-\int_{\Omega}\left(\int_{0}^{j_{o}(x)} S(z) \mathrm{d} z\right) \xi(0, x)
$$

for all test functions $\xi \in L^{p}\left(0, T ; W_{0}^{1, p}(\Omega)\right) \cap L^{\infty}(Q)$ such that $\xi_{t} \in L^{\infty}(Q)$ and $\xi(T, \cdot)=0$, 
(ii) we have

$$
\iint_{\{(t, x) \in Q|M-1 \leqslant| w(t, x) \mid \leqslant M\}} a(w, \nabla w) \cdot \nabla w \rightarrow 0 \quad \text { as } M \rightarrow \infty .
$$

For the homogeneous Dirichlet problem and under various restrictions on the flux $a$, the existence of renormalized solutions is known (see $[2,7,8,16]$ and references therein). Uniqueness for the homogeneous Dirichlet problem is shown in $[7,8,10]$. The theorem below extends these last results to the inhomogeneous case, for fluxes of the form (H0).

THEOREM 7.2. The statement of theorem 2.3 remains true if we assume that $v$ and $\hat{v}$ are renormalized solutions of $\left(P_{g}\left(f, j_{o}\right)\right)$.

Theorem 7.2 follows from the fact that a renormalized solution of $P_{g}\left(f, j_{o}\right)$ is also a weak solution of the problem $\left(P_{g_{M}}^{M}\left(f_{M}, j_{M, o}\right)\right)$ with the corresponding functions $S_{M}, j_{M}, j_{M, o}, \varphi_{M}, g_{M}, F_{M}$ and $f_{M}$ (see $\S 6$ ). Using definition 7.1(ii), we deduce that $f_{M} \rightarrow f$ in $L^{1}(Q)$ as $M \rightarrow \infty$, and then conclude the proof as in $\S 6$.

\subsection{On more general fluxes}

REMARK 7.3. One can allow for a quite general dependency of $a$ on $(t, x)$ when $a$ is Hölder continuous in $w$ of order $\frac{1}{2}$ ( $\left.\underline{\text { cf. }}[1,23]\right)$ or Lipschitz continuous in $w$ (cf. [8]). For less regular convections, the method of doubling of variables remains essential. This method imposes important restrictions on the dependence of $a$ on $x$, especially for the case of non Lipschitz convection. However, one can extend the result of lemma 4.1 and then the ones of theorems 2.3,3.2 to the fluxes of the form

$$
a(t, x, w, \nabla w)=\nabla w+F(w)+G(w) q(t, x)
$$

with $G: \mathbb{R} \rightarrow \mathbb{R}$ continuous and $q: Q \rightarrow \mathbb{R}^{N}$ such that $\operatorname{div}_{x} q=0$ and $q \in L^{\infty}(Q)$ (see [14]).

REMARK 7.4. The same kind of idea gives an approach to the uniqueness for the stationary problem $\left(S_{g}(f)\right)$ in the case of nonlinear diffusion of the form

$$
a(w, \nabla w)=b(\nabla w)+F(w) \quad \text { with monotone continuous } b: \mathbb{R}^{N} \rightarrow \mathbb{R}^{N} .
$$

In this case, one still can obtain the stationary analogue of lemma 4.1 (see, for example, [10]).

In order to avoid the unnecessary complications, let us assume that either the functions $w, \hat{w}$ are bounded, or $b$ is homogeneous (i.e. $b(\lambda \xi)=|\lambda|^{p-1} \lambda b(\xi)$; this includes linear elliptic problems and the $p$-Laplacian). As in lemma 5.2 and theorem 2.3, the $L^{1}$ contraction and comparison property (3.2) would follow, if instead of (5.3) we show that

$$
\limsup _{h \rightarrow 0} \int_{\Omega} \operatorname{sgn}_{0}^{+}(w-\hat{w})(b(\nabla w)-b(\nabla \hat{w}))^{+} \cdot \nabla \xi_{h} \geqslant 0
$$


for an appropriate choice of $\xi_{h}$ satisfying (5.5). To this end, it suffices to assume that

$$
\begin{aligned}
& b=\nabla \Phi \text { for } \Phi \in \mathcal{C}^{2}(\mathbb{R}, \mathbb{R}) \text { with } \\
& \text { the Hessian matrix } D^{2} \Phi \text { satisfying } 1 / \mathcal{M} \leqslant D^{2} \Phi \leqslant \mathcal{M},
\end{aligned}
$$

and replace the auxiliary problem (5.6) in the proof of lemma 5.4 by the appropriate adjoint problem:

$$
\left.\begin{array}{rl}
\operatorname{div}(P(\cdot) \nabla u)=0 & \text { in } \Omega_{h}, \\
u=u_{h}^{o} & \text { on } \partial \Omega_{h}, \\
P=\int_{0}^{1} D^{2} \Phi(\theta \nabla w+(1-\theta) \nabla \hat{w}) \mathrm{d} \theta .
\end{array}\right\}
$$

Indeed, with the notation of the proof of lemma 5.4, the left-hand side of (7.1) can be rewritten as

$$
\int_{\Omega} \nabla(w-\hat{w})^{+} \cdot P(x) \nabla \xi_{h}=\int_{\partial^{\operatorname{int}} \Omega_{h}^{*}}(w-\hat{w})^{+} P(x) \nabla \xi_{h} \cdot n,
$$

which is non-negative, because $\nabla \xi_{h}=\left|\nabla \xi_{h}\right| n$ a.e. on $\partial^{\text {int }} \Omega_{h}^{*}$.

This extends the results of theorem 3.2 to solutions of $\left(S_{g}(f)\right)$ with flux $a$ satisfying $\left(\mathrm{H}^{\prime}\right)$ and $\left(\mathrm{H}^{\prime \prime}\right)$. In the same way we easily obtain the extension of both theorems 2.3 and 3.2 to the case of linear elliptic problems (i.e. for $b(\xi)=A \xi$ with a positive definite matrix $A$ ).

Note that this kind of proof would not work for the evolution problem $\left(P_{g}\left(f, j_{o}\right)\right)$ with nonlinear diffusion, since $\xi_{h}$ would depend on $t$ through $w$ and $\hat{w}$. It should be pointed out that, for the homogeneous Dirichlet problem $\left(P_{0}\left(f, j_{o}\right)\right)$ with flux $a$ of the form $\left(\mathrm{H}^{\prime}\right)$, using the approach of [9], we can prove the $L^{1}$ contraction and comparison principle, provided that $\partial \Omega$ can be locally represented by a graph of a continuous function (see [4]).

REMARK 7.5. As mentioned in remark 5.3, the choice of the distance-to-the-boundary functions

$$
\xi_{h}^{o}=\frac{1}{h} \min \{h, \operatorname{dist}(x, \partial \Omega)\}
$$

readily yields (5.5); then (5.4) follows, by lemma 5.5. Moreover, (5.3) can also be shown, for a sufficiently regular (say, piecewise $\mathcal{C}^{2}$ ) domain $\Omega$. For instance, in the case of a flat portion of the boundary $\{0\} \times U, U \subset \mathbb{R}^{N-1}$, (5.3) with $\xi_{h}=\xi_{h}^{o}$ reduces to the inequality

$$
\frac{1}{h} \int_{0}^{h}\left(\int_{U} \frac{\partial}{\partial x_{N}}(w-\hat{w})^{+} \mathrm{d} x_{1} \cdots \mathrm{d} x_{N-1}\right) \mathrm{d} x_{N} \geqslant 0, \quad \text { for a.e. } t \in(0, T) .
$$

This inequality holds, thanks to the Newton-Leibniz formula.

In the same spirit, proving (7.1) for $\xi_{h}=\xi_{h}^{o}$ would permit us to generalize lemma 5.2. Note that in the assumptions $(\mathrm{H} 2)$ and $\left(\mathrm{H}^{\Omega}\right)(\mathrm{ii})$ we have only to replace $H_{0}^{1}(\Omega)$ by $W_{0}^{1, p}(\Omega)$ and substitute the power $p$ for 2 in the corresponding integrands.

Thus, the results of theorems 2.3, 3.2 can be extended to general nonlinear fluxes of the form $\left(\mathrm{HO}^{\prime}\right)$ if the following question is answered positively. For simplicity, we state it in the case of localized flat boundary and the $p$-Laplacian operator. 
Question 7.6. Let $w, \hat{w} \in W^{1, p}\left(\mathbb{R}^{+} \times \mathbb{R}^{N-1}\right)$ with coinciding traces on $\{0\} \times \mathbb{R}^{N-1}$ and compact support. Denote by $e_{1}$ the unit vector $(1,0, \ldots, 0)$.

Is it true that

$$
\lim _{h \rightarrow 0} \frac{1}{h} \int_{0}^{h} \int_{\mathbb{R}^{N-1}} \operatorname{sgn}_{0}^{+}(w-\hat{w})\left(|\nabla w|^{p-2} \nabla w-|\nabla \hat{w}|^{p-2} \nabla \hat{w}\right) \cdot e_{1} \geqslant 0 ?
$$

It should be pointed out that, by the results of [12], we can assume that a trace of $\operatorname{sgn}_{0}^{+}(w-\hat{w})(b(\nabla w)-b(\nabla \hat{w})) \cdot e_{1}$ on the boundary exists in the weak sense. Indeed, entropy inequalities and an analogue of lemma 4.1 can be obtained with fluxes $\left(\mathrm{H}^{\prime}\right)$. They imply in particular that the distribution $\operatorname{div}_{(t, x)}\left((j(v)-j(\hat{v}))^{+}, \mathcal{F}\right)$ with

$$
\mathcal{F}=\operatorname{sgn}_{0}^{+}(w-\hat{w})(b(\nabla w)-b(\nabla \hat{w}))+\operatorname{sgn}_{0}^{+}(w-\hat{w})(F(w)-F(\hat{w}))
$$

is a Radon measure; furthermore, the two terms in the right-hand side of the above formula can be dissociated, by using the appropriate version of (5.5) and lemma 5.5. For the stationary problem in case $N=1$, this reasoning eventually leads to a positive answer to the above question. Indeed, $\operatorname{sgn}_{0}^{+}(w-\hat{w})\left(b\left(w_{x}\right)-b\left(\hat{w}_{x}\right)\right)$ is a function of bounded variation in this case. Therefore its strong limit exists, as $x \rightarrow 0^{+}$. It is easily seen that this limit cannot be negative, because $b(\cdot)$ is monotone.

\section{References}

1 H. W. Alt and H. W. Luckhaus. Quasilinear elliptic-parabolic differential equations. Math. Z. 183 (1983), 311-341.

2 K. Ammar and P. Wittbold. Existence of renormalized solutions of degenerate ellipticparabolic problems. Proc. R. Soc. Edinb. A 133 (2003), 477-496.

3 K. Ammar, J. Carrillo and P. Wittbold. Scalar conservation laws with general boundary condition and continuous flux function. J. Diff. Eqns 228 (2006), 111-139.

4 B. Andreianov and N. Igbida. Revising uniqueness for a nonlinear diffusion-convection equation. J. Diff. Eqns 227 (2006), 69-79.

$5 \quad \mathrm{Ph}$. Bénilan and H. Touré. Sur l'équation générale $u_{t}=a\left(\cdot, u, \phi(\cdot, u)_{x}\right)_{x}+v$ dans $L^{1}$. II. Le problème d'évolution. Annls Inst. H. Poincaré Analyse Non Linéaire 12 (1995), 727-761.

6 Ph. Bénilan and P. Wittbold. On mild and weak solutions of elliptic-parabolic problems. Adv. Diff. Eqns 1 (1999), 1053-1073.

$7 \quad$ D. Blanchard and F. Murat. Renormalized solutions of nonlinear parabolic problems with $L^{1}$ data: existence and uniqueness. Proc. R. Soc. Edinb. A 127 (1997), 1137-1152.

8 D. Blanchard and A. Porretta. Stefan problems with nonlinear diffusion and convection. J. Diff. Eqns 210 (2005), 383-428.

9 J. Carrillo. Entropy solutions for nonlinear degenerate problems. Arch. Ration. Mech. Analysis 147 (1999), 269-361.

10 J. Carrillo and P. Wittbold. Uniqueness of renormalized solutions of degenerate ellipticparabolic problems. J. Diff. Eqns 156 (1999), 93-121.

11 G. Q. Chen and H. Frid. Divergence-measure fields and hyperbolic conservation laws. Arch. Ration. Mech. Analysis 147 (1999), 89-118.

12 G. Q. Chen and H. Frid. Extended divergence-measure fields and the Euler equations for gas dynamics. Commun. Math. Phys. 236 (2003), 251-280.

13 L. C. Evans and R. F. Gariepy. Measure theory and fine properties of functions, Studies in Advanced Mathematics (Boca Raton, FL: CRC Press, 1992).

14 G. Gagneux and M. Madaune-Tort. Analyse mathématique de modèles non linéaires de l'ingénierie pétrolière. Mathématiques et Applications (Springer, 1996).

15 N. Igbida and J. M. Urbano. Uniqueness for nonlinear degenerate problems. Nonlin. Diff. Eqns Applic. 10 (2003), 287-307. 
16 N. Igbida and P. Wittbold. Renormalized solution for nonlinear degenerate problems: existence and uniqueness. Preprint.

17 K. Kobayasi. The equivalence of weak solutions and entropy solutions of nonlinear degenerate second-order equations. J. Diff. Eqns 189 (2003), 383-395.

18 S. N. Kruzhkov. First order quasilinear equations with several space variables. Mat. USSR Sb. 10 (1970), 217-242.

19 J. Málek, J. Nečas, M. Rokyta and M. Rǔžička. Weak and measure-valued solutions to evolutionary PDEs. Applied Mathematics and Mathematical Computation, vol. 13 (Chapman and Hall, 1996).

20 C. Mascia, A. Porretta and A. Terracina. Nonhomogeneous Dirichlet problems for degenerate parabolic-hyperbolic equations. Arch. Ration. Mech. Analysis 163 (2002), 87-124.

21 A. Michel and J. Vovelle. Entropy formulation for parabolic degenerate equations with general Dirichlet boundary conditions and application to the convergence of FV methods. SIAM J. Numer. Analysis 41 (2003), 2262-2293.

22 F. Otto. Ein Randwertproblem für skalare Erhaltnungssätze. PhD thesis (Universität Bonn, 1993).

23 F. Otto. $L^{1}$ contraction and uniqueness for quasilinear elliptic-parabolic equations. J. Diff. Eqns 131 (1996), 20-38.

24 E. Rouvre and G. Gagneux. Formulation forte entropique de lois scalaires hyperboliquesparaboliques dégénérées. Ann. Fac. Sci. Toulouse Math. 10 (2001), 163-183.

25 F. Simondon. Étude de l'équation $\partial_{t} b u-\operatorname{div} a(b u, \nabla u)=0$ par la méthode des semi-groupes dans $L^{1}$. Publ. Math. Besançon. Analyse Non Linéaire 7 (1983), 1-18.

26 W. P. Ziemer. Weakly differentiable functions: Sobolev spaces and functions of bounded variation. Graduate Texts in Mathematics, vol. 120 (Springer, 1989).

(Issued Publication date 2007) 
